\title{
A spectroscopic analysis of the metallic-line star 2 Ursae Majoris (A2m)
}

\author{
İpek H. Çay \\ Istanbul University, Science Faculty, Department of Astronomy \& Space Sciences, \\ 34119, Istanbul, TURKEY \\ email: ipek@istanbul.edu.tr
}

\begin{abstract}
I derived the elemental abundances of metallic-line star $2 \mathrm{UMa}$, using high-dispersion, high signal-to-noise ratio spectra obtained from Dominion Astrophysical Observatory. This study used Kurucz's ATLAS9 and WIDTH9 programs. The star was selected as 1) it has not been the subject of a detailed elemental abundances analysis, and 2) it is relatively sharp-lined and hot enough so that the continuum can be well placed even in the blue. For even cooler Am stars determining the continium will be a major problem. As a guide for the broad-lined and cooler Am stars, a spectral line atlas of 2 UMa will be prepared.
\end{abstract}

Keywords. Stars: abundances, stars: chemically peculiar, stars: individual: (2 UMa)

\section{Why study 2 UMa?}

2 Ursae Majoris (HD 72037) is a metallic-line star of spectral type A2. This star has not been the subject of a detailed elemental abundances analysis, is relatively sharp-lined, and is hot enough so that the continuum can be well located in the blue. For even cooler Am stars finding the continuum will be a major problem. Once the continuum has been well determined then the abundance analysis can yield reliable results. In the sharplined stars it is possible to measure the weak lines and derive good quality elemental abundances. If the line identifications and elemental abundance analysis of 2 UMa are done very well, it may serve as a guide for other similar Am stars. Well determined abundances can be used for check the Am star theory. As a guide for broad-lined and cooler Am stars, a spectral line atlas of 2 UMa will be prepared.

\section{Data description}

Table 1.

\begin{tabular}{ll}
\hline Observatory & Dominion Astrophysical Observatory \\
Instrument & 1.22 -m. Coudè spectrograph \\
Detectors & RETICON, SITe2, SITe4 \\
Wavelength Coverage & $3825 \AA-4935 \AA$ (total 14 spectra) \\
Dispersion & RETICON and SITe2: $2.4 \AA \mathrm{mm}^{-1}$ (total 10 spectra) \\
& SITe4: $2.4 \AA \mathrm{mm}^{-1}(4$ spectra) \\
$\mathrm{S} / \mathrm{N}$ & $>200$ \\
\hline
\end{tabular}




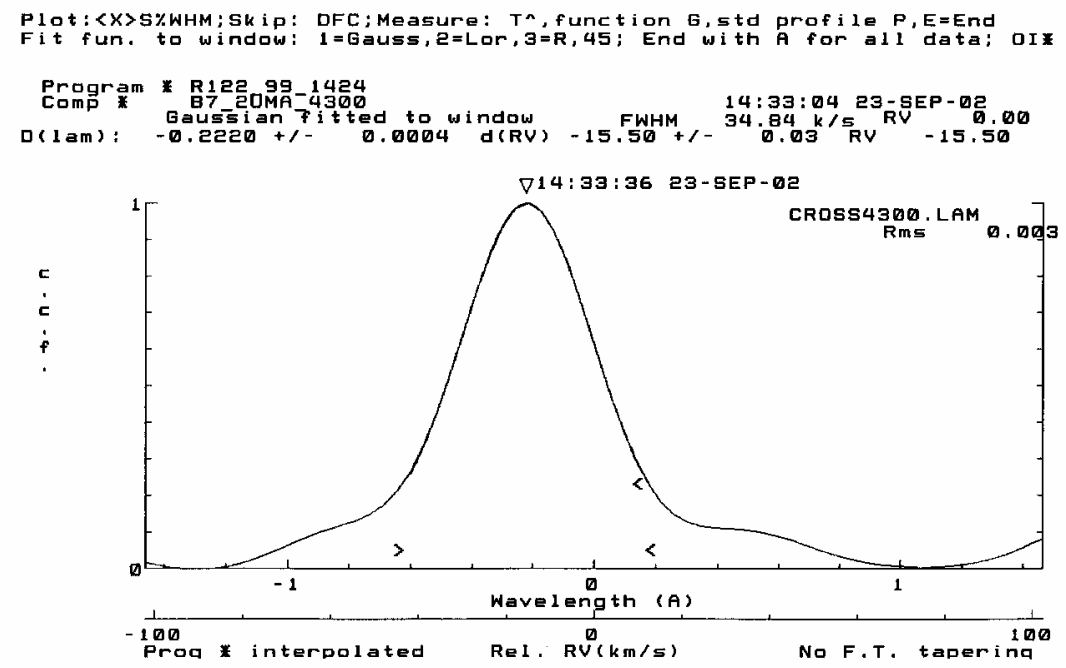

Figure 1. A Sample of radial velocity measurement using VCROSS.

\section{Measurements}

The continuum fitting, radial velocity measurements and equivalent width measurements have been done using the REDUCE (An Interactive Computer Graphics Program) (Hill \& Fisher 1986) package.

\subsection{Radial velocities}

The radial velocities of the spectra have been obtained by cross-correlating each observed spectrum with a synthetic spectrum calculated for the same wavelength range. The synthetic spectra have been calculated using the program SYNTHE (Kurucz \& Avrett 1981). The cross-correlation was achieved with the program VCROSS (Hill \& Fisher 1986). The mean radial velocity from all spectra is $-17.04 \pm 0.04 \mathrm{~km} \mathrm{~s}^{-1}$ (see Figure 1).

\subsection{Continuum fitting and normalization}

Continuum fitting and normalization of each spectrum were made using the program REDUCE. The highest points and adjacent regions in the spectrum have been considered for determining the continuum. Then the spectrum has been divided by the spline fit through these points (see Figure 2).

\subsection{Equivalent widths and the first estimate of the rotational velocity}

The equivalent widths have been measured using the program VLINE (Hill \& Fisher 1986). Because of $2 \mathrm{UMa}$ is a slow rotating star, its spectrum has sharp lines. So that, while measuring the equivalent widths, Gaussian profiles have been fit to the lines. The rotational velocity ( $\mathrm{v}$ sin i) has been estimated as $9 \mathrm{~km} \mathrm{~s}^{-1}$ from the FWHM's of the Gaussian fits which are made to the medium-strength and non-blended lines (see Figure 3). 


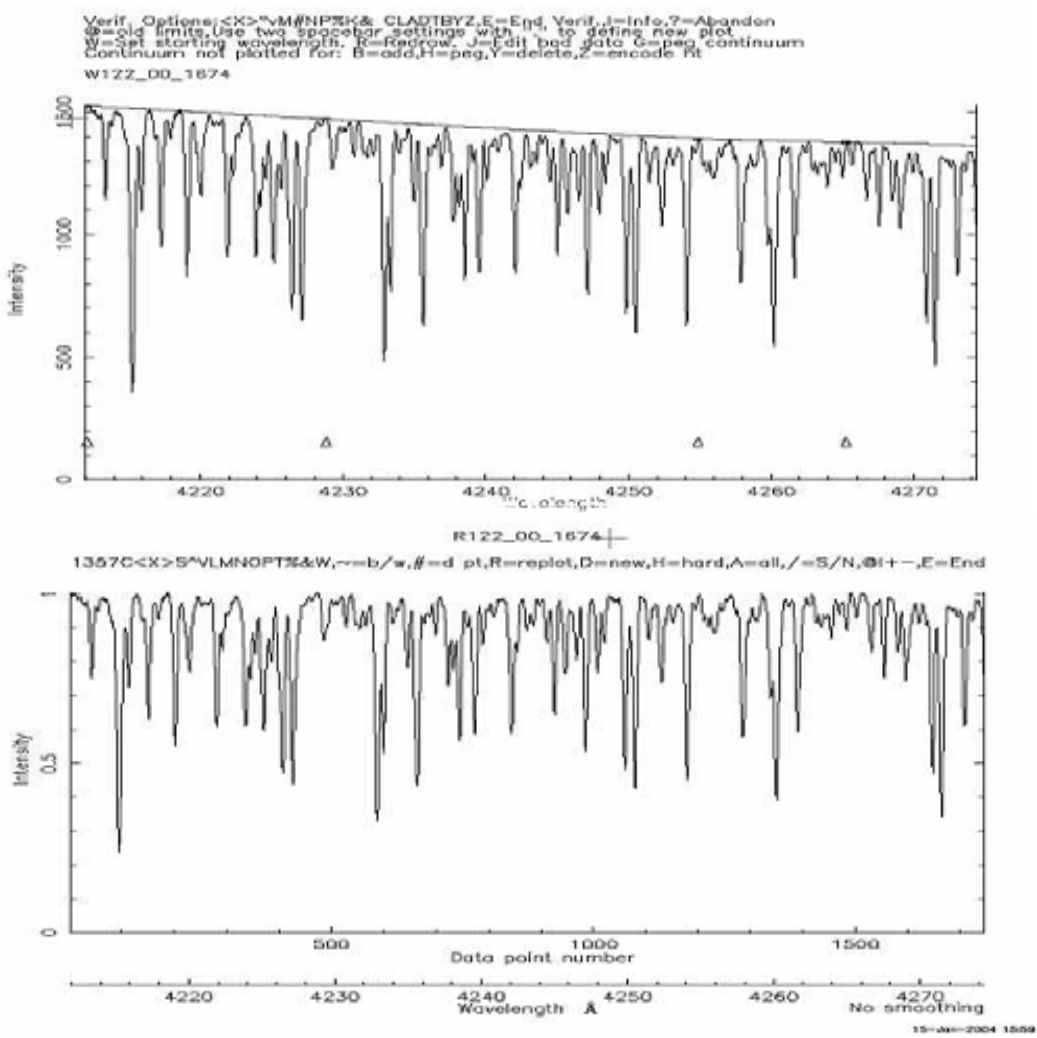

Figure 2. An example of continuum fitting using REDUCE.

Table 2. References used for line identifications to supplement Moore (1945).

\begin{tabular}{ll}
\hline C II, O II & Moore (1993) \\
Si I & Moore (1967) \\
Si II & Moore (1965) \\
S II & Pettersson (1983) \\
Ti II & Huldt et al. (1982), Litzen et al. (2002) \\
V II & Iglesias et al. (1988) \\
Cr I & Kiess (1953) \\
Cr II & Kiess (1951) \\
Mn I & Catalan et al. (1964) \\
Mn II & Iglesias \& Velasco (1964) \\
Fe I & Nave et al. (1994) \\
Fe II & Dworetsky (1971), Johansson (1978), Guthrie (1985) \\
Y II & Nilsson et al. (1991) \\
Ba II & Klose et al. $(2002)$ \\
La II, Ce II, Nd II, & Meggers et al. (1975) \\
Sm II, Eu II, Gd II, & \\
Dy II, Er II, Yb II & \\
\hline
\end{tabular}

\section{Line identifications}

The line identifications of the radial velocity corrected wavelengths were primarily made with the use of the RMT (Moore 1945) and its supplements. Newer line identification tables have been also used for the relevant elements (see Table 2).

Table 3 shows a sample page for line identifications of $2 \mathrm{UMa}$. 
Fix parameter mode

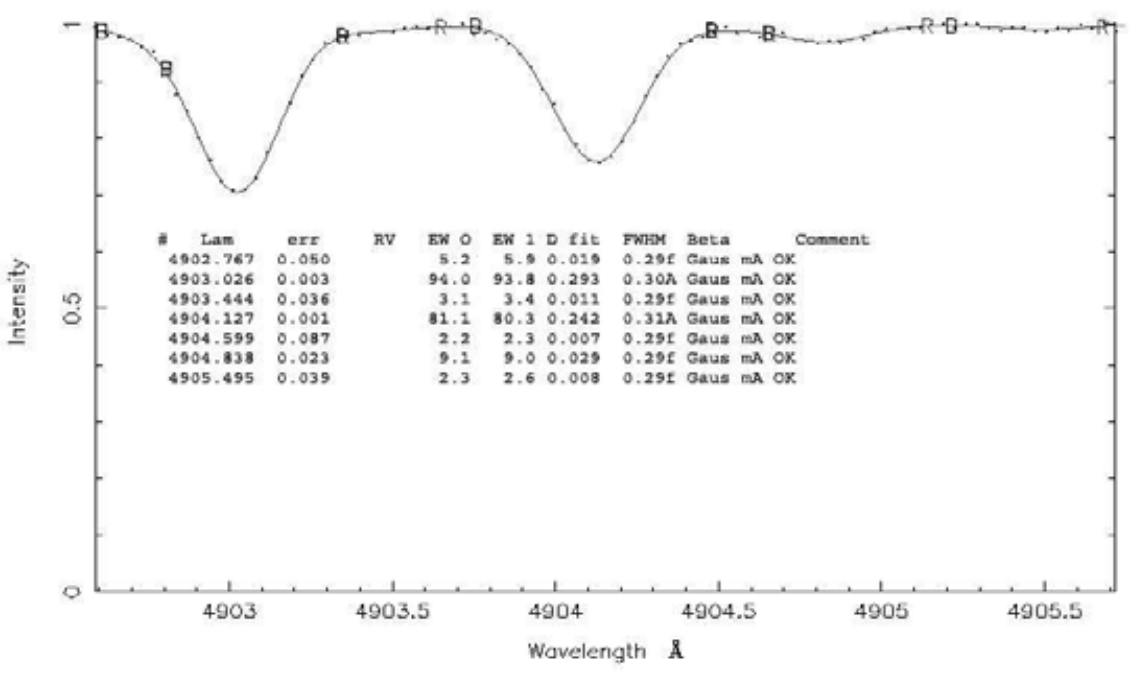

Figure 3. A sample equivalent width measurements using VLINE.

$22-0005-200210150$

Table 3. Sample of line measurements and identifications.

\begin{tabular}{crcccl}
\hline $\begin{array}{c}\text { Measured } \\
\text { Wavelength } \\
(\AA)\end{array}$ & $\begin{array}{r}\text { Equivalent } \\
\text { Width } \\
(\mathrm{m} \AA)\end{array}$ & $\begin{array}{c}\text { Line } \\
\text { Depth }\end{array}$ & $\begin{array}{c}\text { Line } \\
\text { Width } \\
(\AA)\end{array}$ & $\begin{array}{c}\text { Rest } \\
\text { Wavelength } \\
(\AA)\end{array}$ & Identification \\
\hline 3874.490 & 19.6 & 0.079 & 0.23 & 3874.560 & Cr I $(138) 3874.55(75 \mathrm{~W})$ \\
3874.705 & 6.5 & 0.026 & 0.23 & 3874.775 & Ce II(MCS)3874.68(270) \\
3875.039 & 18.2 & 0.074 & 0.23 & 3875.109 & Ce II(MCS)3875.06(140) \\
3875.569 & 11.3 & 0.046 & 0.23 & 3875.639 & (V II (20)3875.656(20)) \\
3875.807 & 14.5 & 0.059 & 0.23 & 3875.877 & Ca I (26)3875.807((4)) \\
3876.007 & 42.8 & 0.173 & 0.23 & 3876.077 & Fe I (22)3876.040(4), \\
& & & & & Ce II(MCS)3876.12(170) \\
3876.692 & 9.8 & 0.039 & 0.23 & 3876.762 & Co I (17,62)3876.831(20) \\
3876.882 & 19.2 & 0.078 & 0.23 & 3876.952 & Ce II (82)3876.97(620) \\
3877.169 & 7.6 & 0.031 & 0.23 & 3877.239 & Pr II(-)3877.18(1700) \\
3877.975 & 190.6 & 0.574 & 0.31 & 3878.045 & Fe I $(20) 3878.0182(60)$ \\
3878.282 & 67.8 & 0.274 & 0.23 & 3878.352 & Y II(7)3878.285(272), \\
& & & & & Ce II(48)3878.36(100) \\
\hline
\end{tabular}

\section{Atmospheric analysis}

\subsection{Determination of the effective temperature and surface gravity}

The initial effective temperature and surface gravity have been estimated as $T_{\text {eff }}=8053$ $\mathrm{K}$ and $\log g=4.15$ with the help of the computer program of Napiwotzi et al. (1993) and the homogeneous uvby $\beta$ data of Hauck \& Mermilliod (1998). These values have been refined to $T_{\text {eff }}=8050 \mathrm{~K}$ and $\log g=4.0$ by comparing the observed $\mathrm{H}_{\beta}$ and $\mathrm{H}_{\gamma}$ profiles to synthetic calculations (see Figure 4). We used ATLAS9 (Kurucz 1993) for calculating the model atmospheres. Our version of ATLAS9 includes the turbulent convection theory of Canuto \& Mazzitelli (1991) which should be more realistic than mixing length theory. We have used the program SYNTHE for calculating the synthetic spectra. 


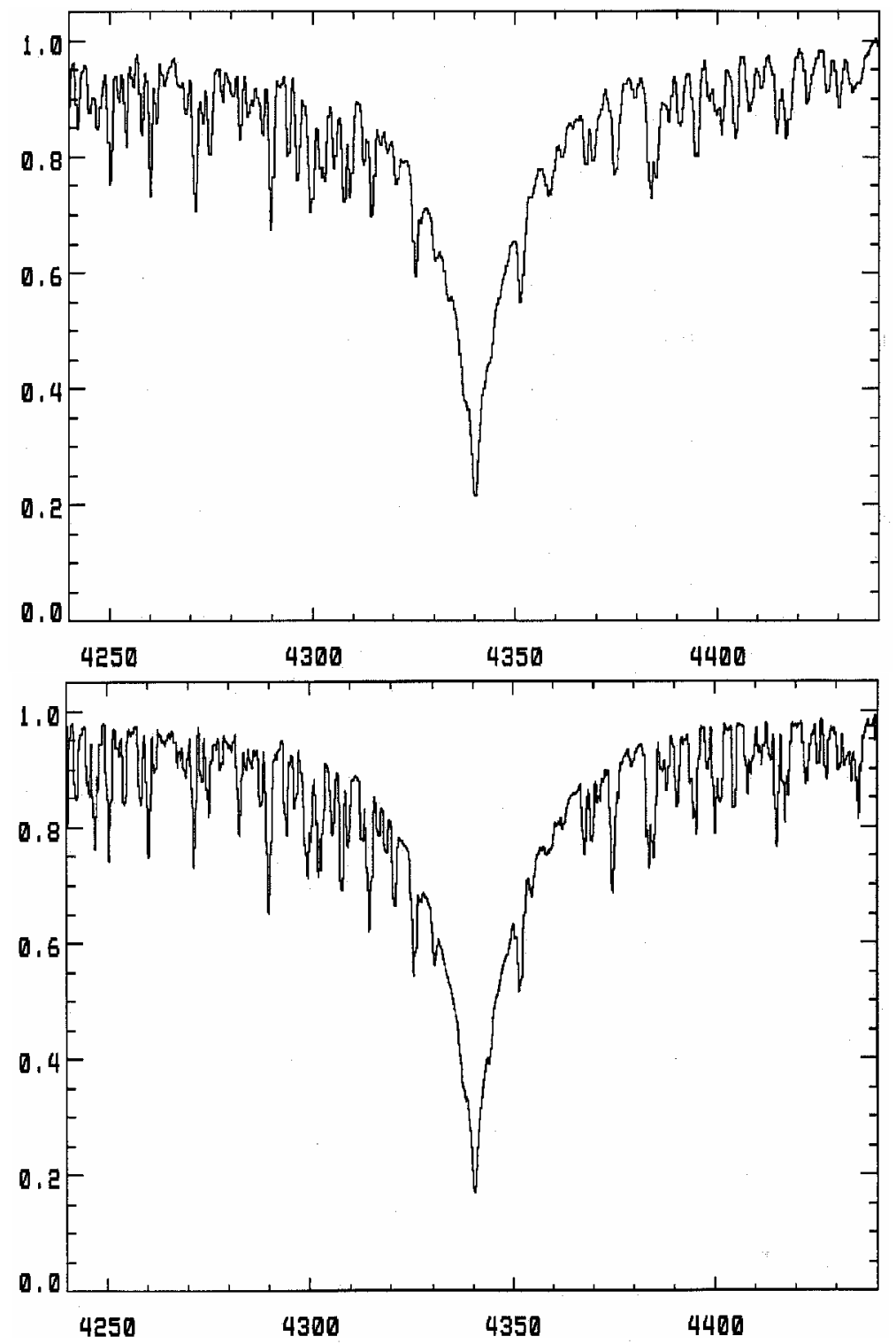

Figure 4. A sample comparison of the observational (top) and the synthetic (bottom) $\mathrm{H}_{\gamma}$ profiles.

\subsection{Determination of the microturbulent velocity and elemental abundances}

The program WIDTH9 (Kurucz 1993) has been used to determine the elemental abundances using the equivalent widths and the atomic data for each line. Abundances from Fe I, Fe II, Cr I and Cr II lines were derived for a range of possible microturbulences. The adopted values (see Table 4) are of which do not show a trend of values for lines of different equivalent widths and have minimal scatter about the mean (see Figure 5).

The abundances of all elements with observed lines have been calculated using the adopted value of the microturbulence. Table 5 compares the derived abundances of 2 UMa with the solar values from Grevesse et al. (1996). 
Table 4. Determination of microturbulence.

\begin{tabular}{lccc}
\hline Species & Number of Lines & $\xi\left(\mathrm{km} \mathrm{s}^{-1}\right)$ & \multicolumn{1}{c}{ ff - values } \\
\hline Fe I & 320 & 2.60 & Fuhr et al. (1988), Kurucz \& Bell (1995) \\
Fe II & 74 & 2.05 & Fuhr et al. (1988), Kurucz \& Bell (1995) \\
& Adopted mean 2.3 & & \\
Cr I & 75 & 2.15 & Martin et al. (1988), Kurucz \& Bell (1995) \\
Cr II & 36 & 2.50 & Martin et al. (1988), Kurucz \& Bell (1995) \\
& Adopted mean 2.3 & & \\
\hline
\end{tabular}

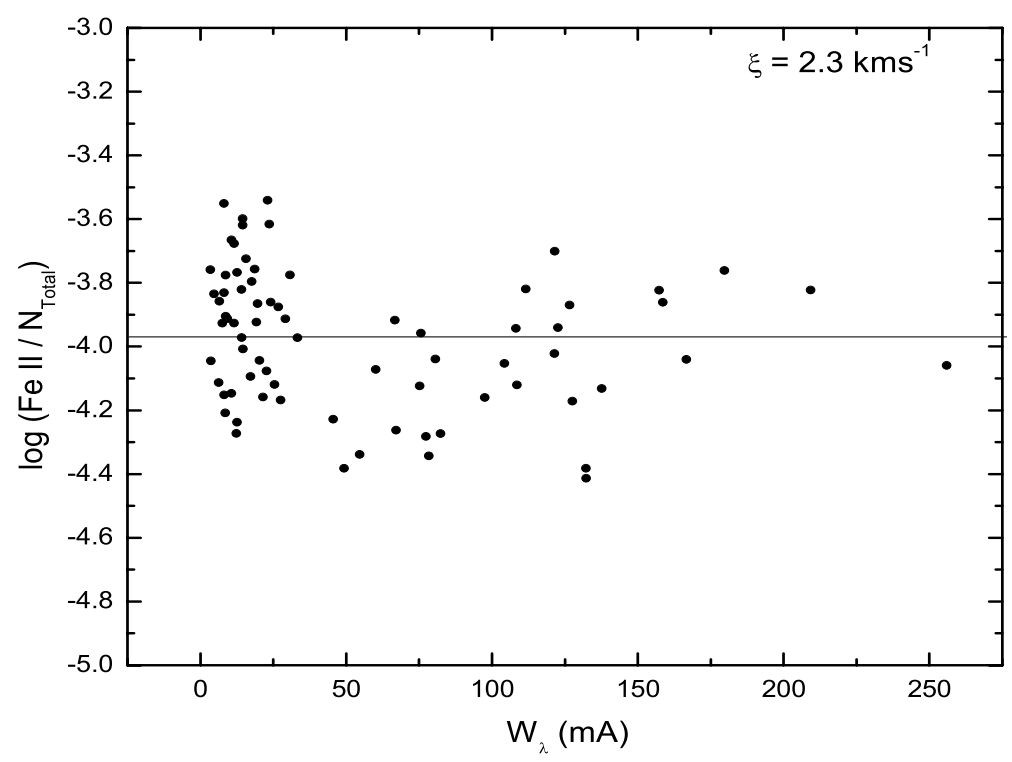

Figure 5. Fe II lines with the minimal scatter about the mean.

\subsection{Determination of the macroturbulence velocity and the final estimate of rotational velocity}

To estimate the macroturbulence and rotational velocity, the calculated abundances have been put into the model atmosphere and a new synthetic spectrum has been calculated to compare to the observed spectrum in $\lambda \lambda 4500-4540$ (see Figure 6 ). Hence, only adjusting the macroturbulence and rotational velocity values, the macroturbulence and final rotational velocity of $2 \mathrm{UMa}$ have been estimated as $5 \mathrm{~km} \mathrm{~s}^{-1}$ and $11 \mathrm{~km} \mathrm{~s}^{-1}$, respectively.

\section{Location of 2 UMa on the HR diagram}

As 2 UMa has a well determined parallax from the HIPPARCOS Satellite (ESA 1997), it is possible to convert its apparent visual magnitude to absolute magnitude and compare with the stellar evolutionary calculations of Schaller et al. (1992) to find its mass and age since the ZAMS. Figure 7 shows the location of 2 UMa on the HR diagram with respect to the mentioned calculations. Using the $T_{\text {eff }}$ value found in this study, the mass of 2 UMa has been estimated as $1.8 \mathrm{M}_{\odot}$ which is the same value found by Kunzli \& North (1998). 
Table 5. Abundances of 2 UMa.

\begin{tabular}{|c|c|c|c|c|}
\hline Species & $\begin{array}{r}\text { Number } \\
\text { of lines }\end{array}$ & $\log (N / H)_{2 \mathrm{UMa}}$ & $\log (N / H)_{\odot}$ & {$[N / H]^{*}$} \\
\hline O I & 1 & -2.44 & -3.13 & 0.69 \\
\hline $\mathrm{Na} \mathrm{I}$ & 1 & -5.03 & -5.67 & 0.64 \\
\hline Mg I & 3 & $-4.89 \pm 0.15$ & -4.42 & -0.47 \\
\hline Mg II & 1 & -4.73 & -4.42 & -0.31 \\
\hline $\mathrm{Al} \mathrm{I}$ & 1 & -4.99 & -5.53 & 0.54 \\
\hline Si II & 4 & $-4.52 \pm 0.23$ & -4.45 & -0.07 \\
\hline S I & 3 & $-4.70 \pm 0.12$ & -4.67 & -0.03 \\
\hline $\mathrm{Ca} \mathrm{I}$ & 15 & $-6.31 \pm 0.19$ & -5.64 & -0.67 \\
\hline Ca II & 1 & -6.52 & -5.64 & -0.88 \\
\hline Sc II & 3 & $-10.28 \pm 0.11$ & -8.83 & -1.45 \\
\hline Ti I & 23 & $-6.76 \pm 0.20$ & -6.98 & 0.22 \\
\hline Ti II & 36 & $-7.12 \pm 0.19$ & -6.98 & -0.14 \\
\hline V I & 6 & $-7.66 \pm 0.04$ & -8.00 & 0.34 \\
\hline V II & 17 & $-7.42 \pm 0.17$ & -8.00 & 0.58 \\
\hline Cr I & 75 & $-5.66 \pm 0.21$ & -6.33 & 0.67 \\
\hline Cr II & 36 & $-5.77 \pm 0.24$ & -6.33 & 0.56 \\
\hline Mn I & 22 & $-6.16 \pm 0.16$ & -6.61 & 0.45 \\
\hline Mn II & 10 & $-6.09 \pm 0.17$ & -6.61 & 0.52 \\
\hline Fe I & 320 & $-3.98 \pm 0.21$ & -4.50 & 0.52 \\
\hline Fe II & 74 & $-3.93 \pm 0.21$ & -4.50 & 0.58 \\
\hline Co I & 16 & $-5.97 \pm 0.21$ & -7.08 & 1.11 \\
\hline $\mathrm{Ni} \mathrm{I}$ & 62 & $-4.91 \pm 0.18$ & -5.75 & 0.85 \\
\hline Ni II & 2 & -4.95 & -5.75 & 0.80 \\
\hline Zn I & 2 & -6.61 & -7.40 & 0.79 \\
\hline Sr I & 1 & -7.21 & -9.03 & 1.82 \\
\hline Sr II & 2 & -8.00 & -9.03 & 1.03 \\
\hline Y II & 9 & $-8.63 \pm 0.19$ & -9.76 & 1.13 \\
\hline Zr II & 19 & $-8.72 \pm 0.19$ & -9.40 & 0.68 \\
\hline Ba II & 2 & -7.78 & -9.87 & 2.09 \\
\hline La II & 32 & $-9.28 \pm 0.20$ & -10.83 & 1.55 \\
\hline Ce II & 111 & $-9.03 \pm 0.20$ & -10.42 & 1.39 \\
\hline Nd II & 23 & $-9.45 \pm 0.22$ & -10.50 & 1.05 \\
\hline Sm II & 6 & $-9.46 \pm 0.20$ & -10.99 & 1.53 \\
\hline Eu II & 3 & $-10.22 \pm 0.12$ & -11.49 & 1.27 \\
\hline Gd II & 1 & -9.58 & -10.88 & 1.30 \\
\hline Dy II & 1 & -9.94 & -10.86 & 0.92 \\
\hline Er II & 2 & -10.09 & -11.07 & 0.98 \\
\hline Yb II & 1 & -9.94 & -10.92 & 0.98 \\
\hline
\end{tabular}

\section{Discussion}

\subsection{Rotational velocity}

Table 6 summarizes the rotational velocity $(v \sin i)$ studies of 2 UMa found in the literature. Fekel gives the same value as in this study. The differences between this and the other studies are mostly due to their lower dispersion spectra. Because of the high dispersion and the high signal-to-noise spectra used in this study, my rotational velocity should be more reliable.

\subsection{Elemental abundances}

Since there is no other detailed elemental abundance analysis of $2 \mathrm{UMa}$ in the literature, Table 7 shows only some values from different studies to compare with this study. Cowley 

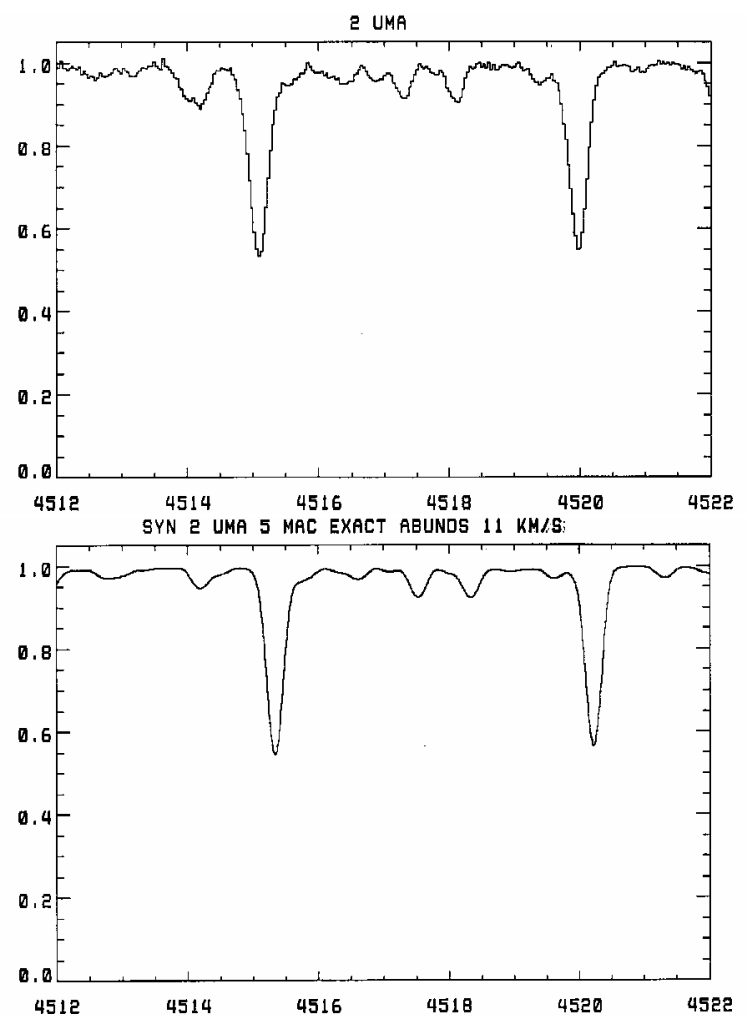

Figure 6. A sample comparison of observational (top) and synthetic (bottom) spectra to determine the macroturbulence and the rotational velocity.

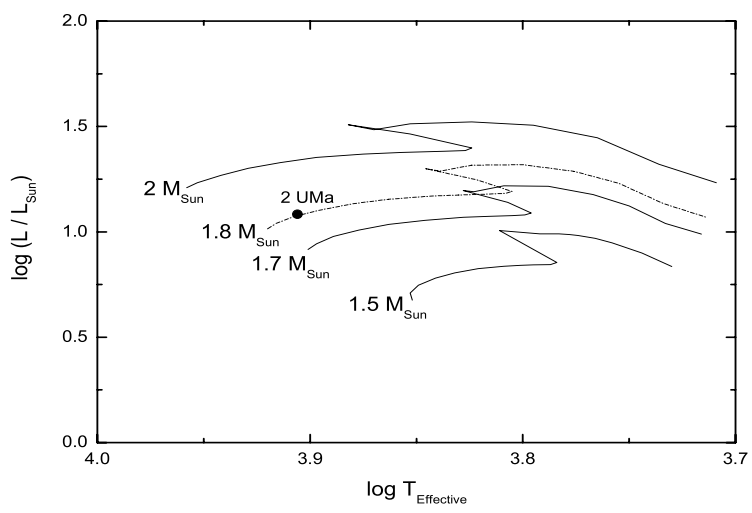

Figure 7. Location of $2 \mathrm{UMa}$ on the HR diagram

\& Aikman (1980) used their own calibration formulae based on the line statistics to derive the abundances and as they noted their method does not give very accurate results. Guthrie (1987) used Ca II K line photometry to obtain the equivalent widths and calculated the Ca II abundance using a differential analysis method. Kunzli \& North (1998) used spectrum synthesis techniques while Adelman et al. (2000) fine analysis techniques similar to this study.

Compared with the solar values, 2 UMa shows considerable underabundances of $\mathrm{Ca}$ and $\mathrm{Sc}$, as expected for an Am star. $\mathrm{Mg}$ is underabundant while Ti, Si and S show nearly 
Table 6. $\mathrm{v}$ sin i determinations.

\begin{tabular}{lc}
\hline Reference & $v \sin i\left(\mathrm{~km} \mathrm{~s}^{-1}\right)$ \\
\hline Abt \& Moyd (1973) & $<10$ \\
Uesugi \& Fukuda (1982) & 15 \\
Hoffleit (1982) & 17 \\
Abt \& Morrell (1995) & 18 \\
Royer et al. (2002) & 26 \\
Fekel (2003) & 11 \\
This Study & 11 \\
\hline
\end{tabular}

Table 7. Comparison of abundance determinations.

\begin{tabular}{lcc}
\hline & Cowley \& Aikman (1980) & This Study \\
Cr II & -6.5 & $-5.77 \pm 0.24$ \\
Mn II & -6.5 & $-6.09 \pm 0.17$ \\
Fe II & -5.0 & $-3.93 \pm 0.20$ \\
& Guthrie (1987) & This Study \\
Ca II & -6.22 & -6.52 \\
& Kunzli \& North (1998) & This Study \\
Ca II & -6.22 & -6.52 \\
& Adelman et al. (2000) & This Study \\
Co I & -6.35 to -6.94 & $-5.97 \pm 0.21$ \\
& (for Am stars including 2 UMA) \\
& \multicolumn{2}{c}{} \\
\end{tabular}

solar abundances. V, Mn and Fe have been found to be overabundant while Al, Cr, Na, $\mathrm{Zr}, \mathrm{Zn}$ and $\mathrm{Ni}$ shows considerable overabundances. Rare earths are also considerably overabundant as expected.

\section{Acknowledgements}

This poster is the summary of the author's PhD. thesis, completed on February 19, 2004 at the Istanbul University and supervised by Dr. H. Gokmen Tektunali (Istanbul University, Astronomy Dept.) and Dr. Saul J. Adelman (The Citadel, Physics Dept.). I thank Dr. James E. Hesser, Director, Dominion Astrophysical Observatory, Herzberg Institute of Astrophysics, for the observing time used by Dr. Adelman to obtain the spectra I analyzed. This work was supported by the Research Fund of The University of Istanbul, Project number : UDP-313/25052004.

\section{References}

Abt, H.A. \& Moyd, K.I. 1973, ApJ, 182, 809

Abt, H.A. \& Morrell, N.I. 1995, ApJS, 99, 135

Adelman, S.J., Gulliver, A.F. \& Lodèn, L.O. 2000, A\&A, 353, 335

Canuto, V. M. \& Mazzitelli, I. 1991, ApJ, 370, 295

Catalan, M.A., Meggers, W.F. \& Garcia - Riquelme, O. 1964, JRNBS, 68A, 9

Cowley, C.R. \& Aikman, G.C.L. 1980, ApJ, 242, 684

Dworetsky, M.M. 1971, Thesis (PhD), - University of California Los Angeles, Source: Dissertation Abstracts International, Vol: 32 - 10 Section:B, p. 5594

ESA 1997, The Hipparcos Tycho Catalogs, SP - 1200

Fekel, F.C. 2003, PASP, 115, 807

Fuhr, J. R.. Martin, G.A., \& Wiese, W.L. 1988, J. Phys. Chem. Ref. Data, 17, Suppl. 4

Grevesse, N., Noels, A. \& Sauval, A.J. 1996, in Holt, S., Sonneborn, G. eds., Cosmis Abundances, ASP Conf.Ser., Vol. 99 Astron.Soc.Pac., San Francisco, p. 117 
Guthrie, B.N.G. 1985, MNRAS, 216, 1

Guthrie, B.N.G. 1987, MNRAS, 226, 361

Hauck, B. \& Mermilliod, M. 1998, A\&AS, 129, 431

Hill, G. \& Fisher, W.A. 1986, Publ.Dom.Astrophys.Obs.Victoria, 16, 27

Hoffleit, D. 1982, The Bright Star Catalogue, Fourth Revised Edition, Yale University Observatory, USA

Huldt, S., Johansson, S., Litzen, U. \& Wyrat, J.-F. 1982, Phys.Scr., 25, 401

Iglesias, L. \& Velasco, R. 1964, Publ. del Instituto de Optica de Madrid, No 23

Iglesias, L., Cabeza, M.I. \& De Luis, B. 1988, Publ. del Instituto de Optica de Madrid, No 47

Johansson, S. 1978, Phys. Scr., 18, 217

Kiess, C.C. 1951 , JRNBS, 47, 385

Kiess, C.C. 1953 , JRNBS, 51, 247

Klose, J.Z., Fuhr, J.R. \& Wiese, W.L. 2002, J. Phys.Chem. Ref. Data, 31, No 1, 217

Kunzli, M. \& North, P. 1998, A\&A,330, 651

Kurucz, R.L. \& Avrett, E.H. 1981, SAO Spec. Rep., No 391

Kurucz, R.L. 1993, Kurucz CD-Rom, No. 13, ATLAS9 Stellar Atmosphere Programs and 2 km/s grid, Smithsonian Astrophysical Observatory, Cambridge, MA

Kurucz, R.L., Bell, B. 1995, Kurucz CD-Rom, No. 23, Atomic Line List, Smithsonian Astrophysical Observatory, Cambridge, MA

Litzen, U. 2002, unpublished

Martin, G.A., Fuhr, J.R. \& Wiese, W.L. 1988, J. Phys. Chem. Ref. Data, 17, Suppl.3

Meggers, W.F., Corliss, C.H. \& Scribner, B.F. 1975, Tables of Spectral-line Intensities. US Government Printing Office, Washington, DC

Moore, C.E. 1945, A Multiplet Table of Astrophysical Interest. Princeton University Observatory, Princeton, NJ

Moore, C.E. 1965, NSRDS - NBS 3, Section 1. US Government Printing Office, Washington, $D C$

Moore, C.E. 1967, NSRDS - NBS 3, Section 2. US Government Printing Office, Washington, $D C$

Moore, C.E. 1993, CRC Series in Evaluated Data in Atomic Physics, ed. J.W. Gallagher

Napiwotzki, R., Schönberner, D. \& Wenske, V. 1993, A\&A, 268, 653

Nave, G., Johansson, S., Learner, R.C.M., Thorne, A.P. \& Brault, J.W. 1994, ApJS, 94, 221

Nilsson, A.E., Johansson, S. \& Kurucz, R.L. 1991, Phys. Scr., 44, 226

Petterson, J.E. 1983, Phys. Scr., 28, 421

Royer, F., Grenier, S., Baylac, M.O., Gomez, A.E. \& Zorec, J. 2002, A\&A, 393, 897

Schaller, G., Schaerer, D., Meynet, G. \& Maeder, A. 1992, A\&AS, 96, 269

Uesugi, A. \& Fukuda, I. 1982, in Proc. of 7th International Codata Conference 